\title{
LA FIXATION DES « CONDITIONS ET LIMITES 》 DU DROIT DE PARTICIPATION EN MATIËRE ENVIRONNEMENTALE EN POLYNÉSIE FRANÇAISE ET EN NOUVELLE-CALÉDONIE
}

\author{
Carine DAVID \\ Maître de Conférences en droit public \\ Habilitée à diriger des recherches (HDR) \\ Université de la Nouvelle-Calédonie \\ Directrice du Master Droit \& Management dans l'environnement calédonien \\ Membre du LARJE
}

Résumé La mise en œuvre de l'article 7 de la Charte de l'environnement relatif au droit à l'information et à la participation du public en matière environnementale a fait l'objet d'une jurisprudence abondante du Conseil constitutionnel, ainsi que d'interventions législatives subséquentes au niveau national. Néanmoins, son application effective en Nouvelle-Calédonie et en Polynésie française pose un certain nombre de difficultés tant s'agissant de la détermination de l'autorité compétente pour intervenir en lieu et place du Parlement pour sa mise en œuvre que de la portée de l'autonomie accordée aux autorités locales dans l'édiction des règles relatives au droit de participation.

Mots clés : Charte de l'environnement, Droit de participation, Nouvelle-Calédonie, Polynésie française, partage de compétence, mise en œuvre, droits fondamentaux.

Summary The determination of " conditions and limits » of the right to participate in environmental matters in French Polynesia and New Caledonia. The implementation of Article 7 of the French Environmental Charter relating to the right to environmental information and public participation was subject to abundant case law of the Constitutional Council, as well as subsequent legislative interventions at national level. However, its effective enforcement in New Caledonia and French Polynesia faces a number of difficulties both regarding the determination of the relevant authority to intervene for its implementation and the scope of autonomy granted to local authorities in issuing rules on the right of participation.

Keywords: French Environmental Charter, Right to public participation, New Caledonia, French Polynesia, power sharing, implementation, fundamental rights. 
La mise en œuvre de l'article 7 de la Charte de l'environnement sur le droit à l'information et à la participation du public en matière environnementale a fait l'objet d'une jurisprudence abondante du Conseil constitutionnel, ainsi que d'interventions législatives subséquentes. Néanmoins, si les décisions du Conseil constitutionnel s'imposent sur le fond aux collectivités situées outre-mer, tel n'est pas le cas de la législation adoptée par le Parlement français lorsque ces collectivités se sont vues transférer la compétence en matière de droit de l'environnement. Or, s'agissant de telles collectivités, certaines interrogations sont apparues s'agissant de l'autorité compétente pour mettre en œuvre le droit de participation, ainsi que de l'encadrement que celle-ci peut prévoir.

En effet, l'article 7 de la Charte, en disposant que "Toute personne a le droit, dans les conditions et les limites définies par la loi, d'accéder aux informations relatives à l'environnement détenues par les autorités publiques et de participer à l'élaboration des décisions publiques ayant une incidence sur l'environnement ", renvoie au législateur le soin d'encadrer ce droit fondamental. Si cela ne pose pas de difficulté sur la plus grande partie du territoire national, tel n'est pas le cas en Nouvelle-Calédonie ou en Polynésie française. En effet, bien que la question ne s'y pose pas dans les mêmes termes, la détermination de l'autorité compétente pour intervenir en lieu et place du Parlement n'est pas évidente (I). Au-delà, des interrogations quant à la portée de l'autonomie accordée à l'autorité locale dans la mise en œuvre du droit de participation sont également prégnantes, notamment eu égard à la décision n²013-308 QPC du 26 avril 2013 relative au Code minier de Nouvelle-Calédonie (II).

\section{LA DÉTERMINATION DE L'AUTORITÉ COMPÉTENTE POUR FIXER « LES CONDITIONS ET LIMITES » DU DROIT DE PARTICIPATION EN POLYNÉSIE FRANÇAISE ET EN NOUVELLE-CALÉDONIE}

La détermination de l'autorité compétente pour fixer «les conditions et limites » de l'article 7 de la Charte de l'environnement en Polynésie française et en NouvelleCalédonie ne se pose pas exactement dans les mêmes termes. Alors que l'Assemblée de la Polynésie française, compétente pour réglementer la matière environnementale, ne dispose pas d'un pouvoir législatif, le Congrès de la NouvelleCalédonie, doté pour sa part d'un tel pouvoir, n'est que partiellement compétent en ce domaine, la compétence de droit commun étant dévolue aux trois provinces, subdivisions territoriales de la Nouvelle-Calédonie.

À partir de ce cadre institutionnel hétérogène, deux questions semblent émerger : le renvoi par le Constituant à la loi pour fixer les conditions et limites du droit de participation implique-t-il dans ces territoires nécessairement l'intervention d'une norme de nature législative? Quelles sont les modalités de mise en œuvre de ce droit directement issu de la Constitution?

RJ・E 4/2015 


\section{A) LA QUESTION DE LA NÉCESSITÉ D'UN ACTE LÉGISLATIF POUR FIXER « LES CONDITIONS ET LIMITES » POUR LA MISE EN CEUVRE DU DROIT DE PARTICIPATION}

Le renvoi par le Constituant au législateur pour fixer «les conditions et limites » du droit de participation impose d'envisager tout d'abord l'éventualité d'une intervention du législateur national pour ce faire en Nouvelle-Calédonie et en Polynésie française. Après avoir écarté cette possibilité, nous examinerons comment la notion de " loi » utilisée par le Constituant peut être appréhendée dans le cadre institutionnel particulier de ces territoires.

\section{L'éventualité de la compétence du législateur national pour mettre en œuvre l'article 7 de la Charte de l'environnement}

La question d'une compétence de l'État pour mettre en œuvre le droit de participation du public en matière environnementale en Polynésie française et en Nouvelle-Calédonie peut paraître surprenante dans la mesure où, dans ces territoires autonomes, l'État est dessaisi de la compétence environnementale au profit des autorités locales. Elle ne se pose donc que dans la mesure où l'on considère que la mise en œuvre d'une disposition constitutionnelle sur habilitation directe du constituant relève des "garanties des libertés fondamentales ", celles-ci relevant de la compétence exclusive de l'État en vertu du titre XII de la Constitution française et de la jurisprudence du Conseil constitutionnel. En d'autres termes, l'interrogation est la suivante : les " conditions et limites " devant être définies par la loi en application de l'article 7 de la Charte de l'environnement doivent-elle être considérées comme des "garanties des libertés publiques » relevant à ce titre de la compétence exclusive de l'État?

L'obstacle majeur pour répondre à cette question réside dans la difficulté de cerner précisément la notion de " garantie des libertés publiques ". En effet, s'il est clair pour le Conseil constitutionnel que "les conditions essentielles de mise en œuvre des libertés publiques et par suite l'ensemble des garanties que celles-ci comportent " ne peuvent dépendre des décisions des collectivités territoriales ${ }^{1}$ et que la doctrine a pu constater qu'en cette matière prévalait un principe "d'unité législative ", la situation n'est, comme souvent, pas aussi claire s'agissant des collectivités autonomes situées outre-mer. Ainsi, si le Conseil d'État a censuré pour incompétence la possibilité pour une autorité territoriale de réglementer la liberté d'association ${ }^{3}$,

1 Conseil constitutionnel, décision ${ }^{\circ}$ 96-373 DC du 9 avril 1996.

$2 \mathrm{~J} .-\mathrm{F}$. Flauss, "Le principe de l'unité législative dans le droit des libertés publiques », LPA 4 avril 1997, p. 4 ou D. Turpin, "Autonomie statutaire des TOM et protection des libertés républicaines ", LPA, 4 déc. 1996, p. 5.

3 CE, ass., 29 avril 1994, n 119562, Haut-commissaire de la République en Nouvelle-Calédonie c/Province Sud, Rec. p. 205 ; RFD adm. 1994, p. 947, conclusions contraires M. Denis-Linton, Chronique AJDA 1994, p. 499, ou encore Dalloz 1995, jurispr. p. 242, note G. Orfila.

RJ・E 4/2015 
cela n'est pas toujours le cas ${ }^{4}$. Pour certains auteurs, ces divergences de jurisprudence pourraient s'expliquer par "l'existence d'une hiérarchie au sein des libertés publiques et des principes à valeur constitutionnelle. II y a d'un côté des libertés publiques fondamentales ayant rang de principe fondamental reconnu par les lois de la République dont les conditions essentielles ne peuvent être créées que par l'État et de l'autre des libertés qui bénéficient d'une protection constitutionnelle plus atténuée et qui peuvent en conséquence être déléguées aux autorités locales „5.

Mais comme le souligne justement Alain Moyrand, "cette explication qui repose sur le caractère "fondamental » des libertés n'est pas aisée à mettre en œuvre car les acceptions de la notion de "droits et libertés fondamentaux » varient selon les auteurs et les juridictions "6, l'auteur concluant que «l'incertitude dans ce domaine est source d'insécuritéjuridique. Les décisions des autorités polynésiennes qui restreignentl'exercice des libertés publiques sont susceptibles d'être déclarées illégales à tout moment "》. Ce raisonnement peut tout à fait être transposé au cas de la Nouvelle-Calédonie.

On le voit, il n'est pas possible de tirer des enseignements clairs de la jurisprudence et de la doctrine en la matière. En tout état de cause, s'agissant de la mise en œuvre de l'article 7 de la Charte de l'environnement, la jurisprudence du Conseil constitutionne ${ }^{\mathbf{8}}$, et plus récemment du Conseil d'État ${ }^{9}$, permettent de considérer que les autorités locales en Polynésie française et en Nouvelle-Calédonie sont compétentes pour fixer les conditions et limites au droit de participation.

\section{La question de la nécessité d'une norme législative locale pour mettre en œuvre le droit de participation}

L'article 7 de la Charte renvoyant au législateur pour fixer l'encadrement du droit de participation du public aux décisions ayant une incidence sur l'environnement, il convient de s'interroger sur la compatibilité de cette règle avec le statut institutionnel de ces collectivités et plus particulièrement avec la nature de leur pouvoir normatif respectif.

En effet, si les assemblées de Polynésie française et de Nouvelle-Calédonie disposent toutes deux du pouvoir d'adopter des "lois du pays ", I'homonymie ne doit

4 Ainsi, CE, sect., 13 mai 1994, n 106608 et 112409, Président de l'assemblée territoriale de la Polynésie française, Rec. p. 234 ; AJDA 1994, p. 559, chron. C. Maugüé et L. Touvet, p. 499.

5 C. Maugüé, L. Touvet, Chronique, AJDA 1994, p. 500. Voir aussi J.-M. Blanquer, «Bloc de constitutionnalité ou ordre constitutionnel ", in Mélanges Jacques Robert, Montchrestien 1998, p. 234 ; F. Garde, Les institutions de la Nouvelle-Calédonie, L'Harmattan, 2001, p. 124.

6 A. Moyrand, Droit institutionnel de la Polynésie française, éd. L'Harmattan, Coll. Perles Océanes, $2^{e}$ édition, 2012, p. 432.

7 lbid.

8 Conseil constitutionnel, Décision n²013-308 QPC du 26 avril 2013, Code minier de Nouvelle-Calédonie.

9 Conseil d'État, 13 février 2015, 10e et 9e sous sections réunies, nº 384447.

RJ・E 4/2015 
toutefois pas laisser penser que ces deux collectivités sont dotées d'un pouvoir de même nature. En effet, alors que la Nouvelle-Calédonie dispose d'un véritable pouvoir législatif, tel n'est pas le cas de la Polynésie française ${ }^{10}$.

La nature juridique de la loi du pays calédonienne n'a fait l'objet que de peu de débats dans la doctrine ${ }^{11}$, tant les intentions des signataires de l'Accord de Nouméa et du Constituant semblaient claires. D'ailleurs, le Conseil constitutionnel|12 et, à sa suite, le tribunal administratif de Nouvelle-Calédonie ${ }^{\mathbf{1 3}}$, la Cour administrative d'appel de Paris ${ }^{\mathbf{1 4}}$ et enfin, le Conseil d'État ${ }^{15}$, n'ont pas hésité à reconnaître la nature législative de la loi du pays calédonienne. À cet égard, l'extension de la procédure de la question prioritaire de constitutionnalité à la loi du pays calédonienne par la loi organique $n^{\circ} 2009-1523$ du 10 décembre 2009 relative à l'application de l'article 61-1 de la Constitution clôt toute discussion quant à la nature législative de la loi du pays calédonienne.

La nature juridique des " actes dénommés » "loi du pays » " adoptés par l'Assemblée de la Polynésie française est différente. En effet, si de manière contestable, le législateur organique a décidé d'opter pour une même appellation ${ }^{\mathbf{1 6}}$, les lois du pays polynésiennes sont en réalité des actes réglementaires que seules des modalités de contrôle spécifiques différencient de la délibération ordinaire. Les guillemets systématiquement utilisés pour désigner cet outil normatif témoignent d'ailleurs de son ambivalence ${ }^{\mathbf{1 7}}$. À cet égard, l'exposé des motifs de la loi organique portant statut d'autonomie de la Polynésie française ${ }^{\mathbf{1 8}}$ qualifie le pouvoir normatif de l'assemblée territoriale de pouvoir " quasi-législatif » lorsqu'il intervient dans le domaine de l'article 34 de la Constitution. Alors que tout semblait destiner la Polynésie française à obtenir un instrument normatif équivalent à celui de la Nouvelle-Calédonie, elle ne dispose finalement que d'un

10 Voir à ce sujet: Carine David, Essai sur la loi du pays calédonienne - La dualité de la source législative dans l'État unitaire français, ed. L'Harmattan, coll. GRALE, 2008.

11 Le débat doctrinal a principalement été alimenté par Olivier Gohin. Voir notamment "L'évolution institutionnelle de la Nouvelle-Calédonie ", AJDA 1999, p. 500-514 ou "Lois du pays - Contrôle du Conseil constitutionnel ", AJDA, 2000, p. 252-258.

12 Décision n 99-410 DC du 15 mars 1999, "Loi organique relative à la NouvelleCalédonie », Rec., p. 51.

13 TA Nouvelle-Calédonie, 2 mars 2000, M. David Bensimon, req. n 9900452, inédit.

14 CAA Paris, 20 décembre 2002, M. Cortot, req. n 02PA00451, La lettre de la Cour, $n^{\circ} 48$, janv. 2003, p. 4/5.

15 CE, Section de l'intérieur, avis n 370.002 du 2 mars 2004 sur l'avant-projet de loi du pays relatif aux procès-verbaux de palabres coutumiers, non publié.

16 Carine David, Essai sur la loi du pays calédonienne - La dualité de la source législative dans l'État unitaire français, ed. L'Harmattan, coll. GRALE, 2008.

17 En effet, aussi bien la loi organique statutaire de 2004 relative à la Polynésie française que les décisions de justice relatives aux « lois du pays " polynésiennes prennent soin d'utiliser des guillemets lorsqu'elles évoquent cet outil.

18 Loi organique n 2004-192 du 27 février 2004 portant statut d'autonomie de la Polynésie française.

RJ・E 4/2015 
simple pouvoir réglementaire, soumis à un contrôle juridictionnel spécifique et complexe, dévolu au Conseil d'État en premier et dernier ressort.

Dès lors, si l'on considère que ces collectivités sont compétentes pour mettre en œuvre l'article 7 de la Charte de l'environnement, on doit considérer - au moins s'agissant de la Polynésie française - que cela doit pouvoir se faire en dehors de l'exercice d'un pouvoir formellement législatif. Telle est d'ailleurs la position du Conseil d'État.

Dans un arrêt du 13 février $2015^{19}$, la Haute juridiction a en effet statué sur la compétence de l'Assemblée de Polynésie française pour fixer « les conditions et limites » de l'article 7 de la Charte de l'environnement. Saisi d'une «loi du pays " portant modification du Code de l'aménagement de la Polynésie française, il a en effet considéré "que lorsque l'Assemblée de Polynésie française édicte, par des actes dénommés " lois du pays ", des mesures relevant du domaine de la loi, il lui incombe de définir les conditions et limites dans lesquelles doit s'exercer le droit reconnu à toute personne par l'article 7 de la Charte de l'environnement d'accéder aux informations relatives à l'environnement détenues par les autorités publiques et de participer à l'élaboration des décisions publiques ayant une incidence sur l'environnement». II a ensuite censuré les dispositions litigieuses en estimant que "en adoptant les dispositions contestées sans déterminer les conditions et limites de la participation du public à la procédure d'actualisation des plans de prévention des risques naturels prévisibles, l'assemblée de Polynésie française a méconnu l'étendue de sa compétence au regard des exigences de l'article 7 de la Charte de l'environnement ".

Par cet arrêt, le Conseil d'État lève une incertitude quant à la possibilité de mettre en œuvre l'article 7 de la Charte de l'environnement par la voie réglementaire, malgré le renvoi au législateur formulé par le Constituant. II est vrai qu'une autre solution aurait rendu en pratique impossible l'adoption de dispositions fixant les conditions et limites du droit d'information et de participation en Polynésie française. Pour ce faire, le Conseil d'État retient ici un critère matériel de la loi et non un critère formel. Pour le Conseil d'État en effet, c'est parce que la « loi du pays » polynésienne intervient dans le domaine de la loi nationale qu'il lui est possible de mettre en œuvre les dispositions de l'article 7 de la Charte de l'environnement.

En conséquence, l'arrêt d'Assemblée “Commune d'Annecy » du 3 octobre $2008^{20}$ ou les arrêts plus récents relatifs à la portée de l'article L. 120-1 du Code de l'environnement rendus par le Conseil d'État n'ont pas vocation à s'appliquer en Polynésie française. La règle selon laquelle le pouvoir réglementaire est incompétent pour intervenir dans la détermination des conditions et limites de l'article 7 de la Charte réservés à la loi ne s'y applique pas dans la mesure où elle ne peut qu'intervenir par la voie réglementaire dans le domaine matériel de la loi tel que fixé par la Constitution de 1958. Du fait de l'incom-

19 CE, 13 février 2015, n³84447.

20 CE, Ass., 3 octobre 2008, n²97931, Commune d'Annecy. 
pétence de l'État dessaisi en la matière, toute autre solution aurait pour effet de vider de sa substance l'article 7 de la Charte de l'environnement en Polynésie française.

Reste à déterminer si cette solution est transposable en Nouvelle-Calédonie. La réponse à cette question s'avère complexe.

\section{B) LA QUESTION DE LA TRANSPOSABILITÉ DU CRITÈRE MATÉRIEL DE LA LOI EN NOUVELLE-CALÉDONIE}

La difficulté réside pour la Nouvelle-Calédonie dans le fait que si le Congrès dispose d'un pouvoir formellement législatif, le partage de compétence y est beaucoup plus complexe qu'en Polynésie française du fait d'un niveau de collectivité supplémentaire, celui des provinces, lesquelles disposent justement de la compétence de droit commun ${ }^{21}$ et par conséquent substantielle en droit de l'environnement. Pour bien comprendre la complexité de la situation en Nouvelle-Calédonie, il doit également être souligné que le pouvoir législatif du Congrès de la Nouvelle-Calédonie n'intervient que dans un certain nombre de domaines, limitativement énumérés à l'article 99 de la loi organique statutaire. Contrairement à la situation en vigueur en Polynésie française, le domaine matériel de la loi du pays ne correspond pas nécessairement au domaine de la loi nationale. Ceci a pour conséquence que suivant les domaines de compétence concernés, le Congrès interviendra soit par la voie législative ${ }^{22}$, soit par la voie réglementaire ${ }^{23}$. Élément de complexité supplémentaire, les assemblées de province de Nouvelle-Calédonie disposent, au même titre que l'Assemblée de Polynésie française ou le Congrès de la Nouvelle-Calédonie, d'un pouvoir réglementaire autonome intervenant dans le domaine de la loi nationale et non soumis au pouvoir réglementaire de l'État. Tel est le cas en l'occurrence du droit de l'environnement.

Dès lors, jusqu'à un avis récent du Conseil d'État ${ }^{24}$, il y avait lieu de s'interroger sur la transposabilité à la Nouvelle-Calédonie de la solution définie par le Conseil d'État dans son arrêt du 13 février 2015 relatif à la Polynésie française.

À cet égard, trois jugements récents du Tribunal administratif de Nouvelle-Calédonie et un arrêt de la Cour administrative d'appel de Paris, tous relatifs à une délibération relative à l'importation, la détention et l'usage en Nouvelle-Calédonie des pesticides $^{25}$ suscitaient un certain nombre d'interrogations.

21 Article 20 de la loi organique modifiée n 99-209 du 19 mars 1999.

22 Par exemple, pour le droit minier ou la domanialité publique.

23 Réglementation phytosanitaire ou urbanisme par exemple.

24 Avis du Conseil d'État du 19 mai 2015, n³ 390000, non publié.

25 Délibération n 217 du 14 août 2012 relative aux conditions d'autorisation, d'importation, de détention, de mise sur le marché et d'utilisation des substances et produits phytosanitaires à usage agricole, JONC n 8818 du 27 août 2012, p. 6343 et S. (http://www.juridoc. gouv.nc/juridoc/jdwebe.nsf/joncentry?openpage\&ap=2012\&page=6343) 
Dans trois jugements du Tribunal administratif de Nouvelle-Calédonie du 16 décembre 201426, les associations "Ensemble pour la Planète (EPLP) » et UFC - Que choisir Nouvelle-Calédonie soulevaient, à l'occasion d'un recours pour excès de pouvoir contre plusieurs arrêtés du Gouvernement de la Nouvelle-Calédonie relatifs à des agréments de substances actives et à des homologations de produits phytosanitaires à usage agricole en Nouvelle-Calédonie, une exception d'inconstitutionnalité à l'encontre de la délibération sus évoquée pour non-respect de l'article 7 de la Charte de l'environnement car adoptée sans avoir fait l'objet de mesures d'information et de participation du public, en violation des exigences constitutionnelles.

Le juge administratif, après avoir constaté que la délibération litigieuse constituait « indéniablement une décision publique ayant une incidence sur l'environnement au sens de l'article 7 de la Charte de l'environnement ", en déduit que celle-ci aurait dû faire l'objet d'une participation du public. Or, il constate que «le Congrès de la Nouvelle-Calédonie n'a fixé par aucun texte, ni de manière générale, ni pour le cas particulier de l'élaboration de la délibération litigieuse de telles "conditions et limites", qu'en ne soumettant pas l'élaboration du contenu de cette délibération à une procédure particulière de participation du public qu'il lui appartenait de définir, le Congrès a ainsi posé à ce droit de participation du public une limite qui méconnait les dispositions de l'article 7 de la Charte ".

Une telle décision ne pouvait qu'être saluée dans la mesure où elle sanctionne l'inaction de l'assemblée locale dans la mise en œuvre de l'article 7 de la Charte en la condamnant pour incompétence négative. Ce faisant, le Tribunal administratif de Nouvelle-Calédonie impose à l'assemblée locale l'édiction de dispositions fixant les conditions et limites du droit d'information et de participation en Nouvelle-Calédonie.

Toutefois, deux mois plus tard, la Cour administrative d'appel de Paris vient remettre en cause ce raisonnement. En effet, dans un arrêt du 12 février 2015, la Cour, justement saisie en appel d'un recours pour excès de pouvoir par l'association EPLP contre la délibération litigieuse, décide de ne pas censurer le Congrès de la NouvelleCalédonie. Invoquant le principe d'inapplicabilité directe de l'article 7 de la Charte, le juge d'appel considère comme inopérant le moyen tiré de la méconnaissance du principe de participation. II juge en effet que l'association requérante ne " saurait utilement invoquer les dispositions de l'article 7 de la Charte de l'environnement, lesquelles, eu égard d'une part à leur portée générale et insuffisamment précise et, d'autre part, au renvoi exprès à la compétence du législateur pour définir les conditions et limites du droit de participation du public qu'elles consacrent, ne sont pas d'effet direct „27.

Or, hasard du calendrier, cette décision intervient la veille de l'arrêt du Conseil d'État évoqué plus haut concernant la Polynésie française. La situation juridique soumise à

26 Affaires $n^{\circ}$ 1400148, 1400158, 1400291, 1400292, 1400349, 1400354 du 16 décembre 2014, Associations Ensemble pour la planète et UFC - Que Choisir Nouvelle-Calédonie.

27 Affaire n 13PA03697, 13PA03698, Association Ensemble pour la planète (EPLP). 
la Cour administrative d'appel de Paris doit dès lors être interprétée à la lumière de cet arrêt. Or, la transposition du raisonnement du Conseil d'État au cas de la délibération calédonienne aboutit à une solution contraire à celle retenue par le juge d'appel.

En effet, le Conseil d'État utilise pour la Polynésie française le critère matériel de la loi. Dans le cas de la délibération litigieuse du Congrès de la Nouvelle-Calédonie, son champ matériel intervient bien dans le domaine de la loi nationale puisque son contenu correspond à des dispositions législatives du Code rural ${ }^{\mathbf{2 8}}$. Dès lors, en application de la règle édictée par le Conseil d'État dans son arrêt du 13 février 2015, il y a lieu de considérer que le Congrès est compétent, au même titre que l'Assemblée de Polynésie française, pour fixer par la voie réglementaire les conditions et limites du droit de participation. C'est d'ailleurs ce que confirme le Conseil d'État dans son avis précité du 19 mai 2015.

C'est heureux car si la Cour administrative d'appel n'a fait en réalité qu'appliquer la jurisprudence du Conseil d'État au regard du cadre juridique national29 pour rejeter le moyen du non-respect de l'article 7 de la Charte du fait de l'absence d'effet direct de cette disposition constitutionnelle, la transposition du raisonnement n'est pas tenable dans le contexte juridique particulier de la Nouvelle-Calédonie. En effet, au niveau national, il n'y a pas identité d'autorité compétente pour fixer les conditions et limites de l'article 7 de la Charte et pour appliquer lesdites conditions et limites : le Parlement fixe par une loi les conditions et limites du droit de participation qui s'imposent au Gouvernement et aux autorités locales dans l'exercice de leur pouvoir réglementaire. Dans le cas de la Nouvelle-Calédonie, il y a identité d'autorités compétentes : le Congrès de la Nouvelle-Calédonie est compétent pour fixer les conditions et limites du droit de participation, y compris par la voie réglementaire lorsqu'il intervient dans le domaine de la loi, en application de la jurisprudence du Conseil d'État du 13 février 2015. Le même Congrès de la Nouvelle-Calédonie, toujours par la voie réglementaire, intervient pour mettre en œuvre ces conditions et limites puisque la compétence des autorités locales couvre l'équivalent du domaine de la loi nationale et du décret dérivé ${ }^{30}$.

Dans ces conditions, la solution retenue par la Cour administrative d'appel de Paris permet donc tout simplement au Congrès de la Nouvelle-Calédonie de s'affranchir indéfiniment de la mise en œuvre des droits d'information et de participation dans ses domaines de compétence. Une telle solution n'est évidemment pas acceptable. En ce sens, le raisonnement du Tribunal administratif de Nouvelle-Calédonie, certainement plus au fait des subtilités juridiques locales, concluant à la censure de l'incompétence négative du Congrès de la Nouvelle-Calédonie, constitue l'unique raisonnement envisageable, sauf à vider de sa substance l'article 7 de la Charte de l'environnement en Nouvelle-Calédonie.

28 Articles L. 253-1 et s. du Code rural et de la pêche maritime.

29 Voir, par exemple, CE, $2^{e}$ et $7^{e}$ sous-sections réunies, 4 décembre 2013, 357839 (considérant $\left.n^{\circ} 6\right)$.

30 Ainsi, par exemple, lorsque le Congrès intervient pour réglementer l'utilisation des pesticides en matière agricole, il fixe par délibération les dispositions relevant des parties législative et réglementaire du Code rural. 
Au surplus, il convient de souligner que le raisonnement formulé par la Cour administrative d'appel de Paris est tout à fait transposable aux provinces de NouvelleCalédonie, compétentes pour une part importante du droit de l'environnement. En effet, celles-ci intervenant, comme en Polynésie française, par la voie réglementaire dans le domaine de la loi nationale, il leur suffirait alors de s'abstenir de tout dispositif visant à mettre en œuvre le droit de participation du public pour s'affranchir elles aussi de l'article 7 de la Charte lors de l'adoption des dispositions de leur code de l'environnement respectif. Ceci aurait pour conséquence que l'ensemble des réglementations environnementales n'auraient pas à être soumis au principe de participation du public, seuls les arrêtés d'application devant l'être. C'est dans ce contexte qu'est intervenu l'avis du Conseil d'État en date du 19 mai 2015 sus évoqué.

\section{LA PORTÉE DE LA COMPÉTENCE DES AUTORITÉS LOCALES POUR FIXER LES CONDITIONS ET LIMITES DU DROIT DE PARTICIPATION DU PUBLIC}

Dans cet avis non publié31, le Conseil d'État tranche la question de la répartition des compétences entre l'État, la Nouvelle-Calédonie et les provinces pour définir les conditions de participation du public aux décisions ayant une incidence sur l'environnement. II indique notamment que les collectivités déterminent les conditions et limites de la participation à l'élaboration des décisions publiques ayant une incidence sur l'environnement dans le cadre de leurs compétences respectives.

Dès lors que les autorités locales de Polynésie française et de Nouvelle-Calédonie sont compétentes pour fixer les conditions et limites de l'article 7 de la Charte de l'environnement, il y a lieu de déterminer la portée de ce droit et les modalités de sa mise en œuvre. À cet égard, deux questions se posent. La première est relative au domaine matériel d'intervention des autorités locales (A), la seconde a trait à l'autonomie décisionnelle de ces mêmes autorités locales (B).

\section{A) LE DOMAINE MATÉRIEL D'INTERVENTION DES AUTORITÉS LOCALES}

Là encore, la situation est plus simple en Polynésie française qu'en NouvelleCalédonie, les trois niveaux de collectivité y étant pour beaucoup dans la complexité du questionnement dans ce dernier territoire.

En effet, la Polynésie française est la seule collectivité locale susceptible d'intervenir de manière autonome dans le domaine de la loi, les communes ne disposant que d'un pouvoir réglementaire relevant du droit commun des collectivités territoriales, c'est-à-dire

31 La teneur de cet avis a été rendue publique dans la toute nouvelle revue d'actualité juridique des services de l'État en Nouvelle-Calédonie : D-Codex, Revue d'information juridique et d'actualité institutionnelle, n² 2, septembre 2015.

RJ・E 4/2015 
assujetti au pouvoir réglementaire national. Dès lors, la Polynésie française disposant de l'ensemble des compétences relevant du domaine de la loi, le domaine d'intervention de l'Assemblée de Polynésie française couvre a priori l'ensemble des compétences susceptibles de nécessiter un cadre juridique pour la mise en œuvre du principe de participation.

Tel n'est pas le cas en Nouvelle-Calédonie dans la mesure où le partage de compétences établi par la loi organique statutaire ${ }^{32}$ répartit les domaines susceptibles de nécessiter la fixation d'un cadre juridique pour l'exercice du droit de participation entre la NouvelleCalédonie et les provinces. Ainsi, si la Nouvelle-Calédonie est par exemple compétente en matière de droit minier, de santé publique, de domanialité publique de la NouvelleCalédonie et des provinces, de réglementation et d'exercice des droits d'exploration, d'exploitation, de gestion et de conservation des ressources naturelles biologiques ou non biologiques de la zone économique exclusive ou encore les principes directeurs de droit de l'urbanisme, la réglementation zoosanitaire et phytosanitaire et l'énergie, les provinces interviennent pour leur part en vertu de leur compétence de droit commun pour réglementer des domaines tels que le droit de la nature, le droit des déchets, les installations classées pour la protection de l'environnement ou encore les sites et paysages... ${ }^{33}$

Il y a dès lors lieu de s'interroger sur la pertinence de la solution d'éclatement de la compétence de mise en œuvre de l'article 7 de la Charte de l'environnement préconisée par le Conseil d'État dans son avis du 19 mai 2015. En effet, une telle solution permet à chaque collectivité d'intervenir à son gré en matière de participation dans ses différents domaines de compétence. Ne vaudrait-il pas mieux envisager l'adoption par le législateur calédonien d'une législation cadre? Cette solution aurait d'ailleurs le mérite de répondre à l'exigence fixée par le Constituant à l'article 7 de la Charte de l'intervention d'une loi pour fixer les conditions et limites du droit de participation.

Or, le Conseil d'État se positionne totalement à rebours d'une telle hypothèse en considérant au surplus que les provinces étant compétentes en matière d'environnement ${ }^{34}$, elles peuvent fixer un régime juridique de droit commun, analogue à celui contenu dans les articles L. 120-1 et suivants du Code de l'environnement national, qui serait applicable aux décisions non soumises à une procédure particulière. Pire, le Conseil d'État préconise que ces régimes juridiques provinciaux de droit commun aient vocation à s'appliquer à titre subsidiaire à l'État, à la Nouvelle-Calédonie et aux communes lorsque l'État et la Nouvelle-Calédonie ne définissent pas les conditions et limites de mise en œuvre de l'article 7 de la Charte de l'environnement dans leurs domaines de compétence respectifs.

Dès lors, il apparaît que chaque collectivité a le pouvoir d'édicter, à l'occasion de la mise en place ou de la modification d'une réglementation, des mesures visant à mettre en œuvre les principes d'information et de participation. Ainsi, les provinces

32 Loi organique modifiée n 99-209 du 19 mars 1999 relative à la Nouvelle-Calédonie.

33 Articles 20 et 22 de la loi organique n 99-209 précitée.

34 On vient de voir que cela n'est pas tout à fait exact.

RJ・E 4/2015 
de Nouvelle-Calédonie ont d'ores et déjà inclus dans leur Code de l'environnement respectif des dispositifs relatifs aux droits à l'information et à la participation en matière environnementale, principalement à travers la définition des procédures d'enquêtes publiques. La Province Sud a pour sa part édicté en juin $2015^{35}$ des règles transversales relatives à la mise en œuvre du principe de participation du public en matière environnementale, s'appliquant aux décisions non soumises à une procédure particulière en ce domaine. De son côté, la Nouvelle-Calédonie a également eu l'occasion d'insérer des dispositifs de participation du public dans le cadre de son Code minier.

En conséquence, on ne peut que constater aujourd'hui le caractère irrationnel de la situation ainsi créée dans la mesure où la Province Nord de Nouvelle-Calédonie a adopté un dispositif réglementaire sur l'information et la participation par une délibération du 30 janvier $2015^{36}$ alors que la Province Sud vient d'adopter un mécanisme différent quelques mois plus tard ${ }^{37}$. La troisième province, celle des Îles Loyauté, réfléchit pour sa part à la mise en place d'un dispositif adapté prenant en compte les spécificités induites par la présence quasi exclusive sur son territoire de la population autochtone traditionnelle.

Au surplus, la situation de la Nouvelle-Calédonie ou de l'État devant une telle construction apparaît des plus discutables. En effet, comment appliquer le principe de subsidiarité des régimes de droit commun provinciaux dans l'hypothèse d'une carence du législateur national ou local? Pour expliciter le caractère farfelu de cette solution, il suffit d'appliquer la situation préconisée par le Conseil d'État au cas évoqué plus haut de la délibération litigieuse du Congrès de la Nouvelle-Calédonie sur les pesticides. On se situe en effet bien dans l'hypothèse de carence envisagée par le Conseil d'État dans la mesure où l'assemblée locale n'a pas fixé les conditions et limites du droit de participation dans un de ses domaines de compétence. Dans ce cas, comment déterminer les mesures de participation applicables? Quel régime juridique choisir parmi les trois régimes de droit commun provinciaux? II n'existe aucune raison de faire prévaloir un régime juridique sur un autre dès lors que trois régimes existeront. II ne parait pas non plus envisageable que les modalités de participation du public sur une même décision diffèrent en divers endroits du territoire.

Dans ce contexte, il serait salutaire que la section contentieux du Conseil d'État prenne la mesure de la situation ainsi créée et revienne sur la position émise par la section administrative à l'occasion du recours en cassation contre l'arrêt de la Cour administrative d'appel de Paris du 12 février 2015 évoqué plus haut.

II nous semble que la fixation par une loi du pays d'un cadre général de mise en œuvre de l'article 7 de la Charte, allant au-delà de la compétence matérielle de la

35 Délibération n 17/2015 APS du 26 juin 2015, JONC 2 juillet 2015, p. 5335 et s.

36 Délibération $n^{\circ}$ 2015-39/APN du 30 janvier 2015 modifiant la délibération modifiée $n^{\circ}$ 2008-306/APN du 24 octobre 2008 relative au Code de l'environnement de la province Nord, JONC n 9127 du 19 février 2015, p. 1456 et s.

37 Délibération du 26 juin 2015, ibid.

RJ・E 4/2015 
Nouvelle-Calédonie, serait une solution bienvenue. Elle aurait le mérite de ne pas multiplier les cadres juridiques existant sur le territoire national s'agissant tout de même de la mise en œuvre d'une disposition constitutionnelle!

Cette solution paraitt tout à fait envisageable juridiquement sans qu'il y ait lieu de considérer que la Nouvelle-Calédonie empiète sur les compétences provinciales. En effet, bien que l'article 99 de la loi organique statutaire de 1999 qui fixe le domaine matériel de la loi du pays calédonienne n'évoque pas expressément la possibilité d'intervention du législateur local pour fixer les principes fondamentaux du droit de l'environnement, il est possible de faire application de la jurisprudence du Conseil constitutionnel sur l'extension du domaine de la loi nationale ${ }^{\mathbf{3 8}}$, le juge constitutionnel procédant par ailleurs le plus souvent par assimilation entre les deux instruments législatifs ${ }^{39}$.

Le juge constitutionnel a en effet édifié plusieurs techniques qui ont permis une extension considérable du domaine de la loi au niveau national. Le Conseil constitutionnel estime ainsi possible pour le législateur d'intervenir dans des domaines liés à des matières de l'article 34 de la Constitution. Dans sa décision du 27 juillet 198240, le Conseil constitutionnel a accepté que le législateur intervienne dans des domaines qui, bien que non mentionnés à l'article 34 de la Constitution, n'étaient cependant pas dépourvus de tout lien avec ceux-ci. En effet, le Conseil constitutionnel a précisé que "par son objet même, le contenu du plan national pluriannuel touche à des matières réservées à la loi ». II apparaît donc que le législateur peut être considéré comme compétent dans des matières éloignées des domaines énumérés à l'article 34 de la Constitution, mais qui ne sont néanmoins pas dépourvues de tout lien avec ces derniers. Le domaine de la loi est donc extensible.

L'exemple du droit de l'environnement est tout à fait pertinent à cet égard. En effet, le droit de l'environnement n'a été inséré à l'article 34 de la Constitution que lors de la révision constitutionnelle du $1^{\text {er }}$ mars 2005 alors même que le législateur intervenait depuis bien longtemps dans ce domaine, sous le regard bienveillant du Conseil constitutionnel. II paraît en conséquence plausible que le même Conseil constitutionnel ne voit pas forcément d'objection à la reproduction d'un mouvement similaire à celui ayant eu lieu au niveau national au niveau de la Nouvelle-Calédonie, d'autant qu'il s'agit ici de mettre en œuvre des dispositions constitutionnelles et non de dessaisir les provinces de leur compétence environnementale.

38 Carine David, Essai sur la loi du pays calédonienne - La dualité de la source législative dans l'État unitaire français, ed. L'Harmattan, coll. GRALE, 2008.

39 Carine David, "Commentaire de la décision n²013-308 QPC du 26 avril 2013 - Le difficile positionnement du Conseil constitutionnel par rapport à la loi du pays : entre assimilation et reconnaissance des spécificités ", Revue Française de Droit Constitutionnel, n 95 , 2013/3 et Carine David, «L'assimilation du régime des lois du pays à celui des lois nationales dans le cadre de la QPC », Revue Française de Droit Constitutionnel, n² 2012/4, p. 863-866. 40 Décision n 82-142 DC du 27 juillet 1982, "Réforme de la planification », Rec., p. 52.

RJ・E 4/2015 


\section{B) LA PORTÉE DE L'AUTONOMIE NORMATIVE DES ASSEMBLÉES ULTRAMARINES DANS LA DÉFINITION DES CONDITIONS ET LIMITES DES DROITS D'INFORMATION ET DE PARTICIPATION}

II reste par ailleurs à s'interroger sur la portée matérielle du pouvoir d'encadrement des assemblées ultramarines et particulièrement de l'autonomie qu'elles pourront exprimer dans la mise en œuvre de l'article 7 de la Charte de l'environnement. Cette question s'avère capitale dans la mesure où la Nouvelle-Calédonie comme la Polynésie française sont identifiées comme des " hot spots " 41 de la biodiversité mondiale ${ }^{42}$ et qu'une partie importante du lagon calédonien est inscrit au patrimoine mondial de I'UNESCO. À cela s'ajoute un retard important dans les deux territoires en termes de gestion des déchets, d'assainissement des eaux usées, des énergies renouvelables, de la gestion de l'eau.

Dans ce cadre, le respect des principes fixés par la Charte de l'environnement de 2005 s'avère primordial, particulièrement dans le domaine du droit minier. À cet égard, la décision du Conseil constitutionnel du 26 avril 2013, rendue à l'occasion d'une QPC relative à des dispositions du Code minier de Nouvelle-Calédonie, donne quelques indications. En effet, une association de protection de l'environnement avait soulevé une QPC à l'égard de dispositions législatives ${ }^{43}$ contenues dans le Code minier de Nouvelle-Calédonie ${ }^{44}$, à l'occasion d'un litige portant sur l'attribution par la Province Nord d'un permis de recherche minier à une entreprise de prospection minière. L'association requérante reprochait en effet à la procédure d'attribution d'un tel permis de ne pas respecter l'article 7 de la Charte de l'environnement en ne prévoyant pas de mesures d'information et de participation du public alors même qu'une telle décision a nécessairement un impact environnemental. La décision rendue dans cette affaire démontre les difficultés rencontrées par le juge constitutionnel pour appréhender l'autonomie du législateur calédonien tout en préservant l'unité de sa jurisprudence.

41 Pour être considéré comme un « hot spot », deux éléments sont pris en compte : l'endémisme et le degré de menace qui pèse sur les espèces. La Nouvelle-Calédonie répond incontestablement à ces deux critères avec un taux d'endémisme particulièrement élevé et des menaces caractérisées par une activité minière importante, ses terres contenant un tiers des réserves mondiales de nickel.

42 Malgré une superficie 20 fois moindre que la France, la Nouvelle-Calédonie abrite presque autant d'espèces végétales terrestres. On dénombre aujourd'hui près de 3261 espèces de végétaux vasculaires dont $76 \%$ d'espèces endémiques, ce qui la classe au troisième rang mondial de l'endémisme après Hawaï avec $89 \%$ et la Nouvelle-Zélande avec $82 \%$.

43 Dispositions issues de la loi du pays n²009-6 du 16 avril 2009 au Code minier de NouvelleCalédonie (partie législative), JONC du 21 avril 2009, p. 3007 et plus particulièrement relatives à l'octroi de permis de recherche, en application de la procédure prévue à l'article L. 141-10 du Code.

44 En application du $6^{\circ}$ de l'article 99 de la loi organique du 19 mars 1999, le Congrès de la Nouvelle-Calédonie peut adopter des règles concernant les hydrocarbures, le nickel, le chrome et le cobalt. C'est dans ce cadre que le Congrès a adopté par une loi du pays n²009-6 du 16 avril 2009 le Code minier de la Nouvelle-Calédonie. 
Dans le considérant final de cette décision, le Conseil constitutionnel décide que "le législateur a pu considérer que les autorisations de travaux de recherches ne constituent pas des décisions ayant une incidence significative sur l'environnement; que, par suite, en ne prévoyant pas de procédure d'information et de participation du public préalable à l'intervention des autorisations de travaux de recherches, le législateur a fixé, au principe d'information et de participation du public, des limites qui ne méconnaissent pas l'article 7 de la Charte de l'environnement "45.

Le Conseil constitutionnel impose donc dans cette décision la nécessité d'une incidence " significative » sur l'environnement des décisions en cause pour imposer une information et une participation du public, comme il l'a déjà fait au niveau national ${ }^{\mathbf{4 6}}$.

Le juge constitutionnel considère que le constituant a consenti au législateur une " certaine latitude " en utilisant l'expression "dans les conditions et limites ", qui habilite plus largement ce dernier que s'il s'était contenté de faire référence aux seules « conditions » ou « modalités ».

En faisant le choix d'une assimilation entre législateur local et national alors qu'aucun article du Code minier de Nouvelle-Calédonie ne qualifiait en l'espèce les incidences ou effets sur l'environnement que doivent avoir les décisions prises par l'autorité publique pour faire l'objet d'une information et d'une participation du public, il nous semble que le Conseil fait dans cette décision œuvre législative et se substitue au législateur du pays pour apprécier la portée des conditions et limites qu'il souhaite fixer au droit de participation du public en imposant une lecture de l'article 7 de la Charte à la lumière de l'ancien standard législatif national. Ce faisant, il fait obstacle à une appréhension autonome et plus protectrice de l'environnement du droit de participation par le législateur local ${ }^{47}$.

Notons toutefois que le Conseil a apporté deux limites salutaires quant à la portée de sa décision en considérant que sa décision devait être entendue "compte tenu de la nature des substances minérales susceptibles d'être recherchées et en l'état des techniques mises en cuvre".

D'une part, la décision ne concerne donc que les substances minérales actuellement recherchées en Nouvelle-Calédonie, à savoir le cobalt, le nickel et le chrome. Une telle solution paraît satisfaisante dans la mesure où des gisements de gaz naturel et de pétrole

45 Considérant $n^{\circ} 11$.

46 Décision n² 2012-282 QPC du 23 novembre 2012, Association France Nature Environnement et autres [Autorisation d'installation de bâches publicitaires et autres dispositifs de publicité].

47 Cette assimilation paraît d'autant plus discutable que le législateur national a décidé luimême de ne plus s'imposer de telles limites. En effet, le Parlement a réécrit l'article L. 120-1 en ne qualifiant plus les incidences sur l'environnement, rehaussant ainsi le niveau d'exigence en matière d'information et de participation (loi n² 2012-1460 du 27 décembre 2012). 
auraient été découverts dans les eaux territoriales de la Nouvelle-Calédonie, mais à une profondeur qui ne permettrait pas une exploitation rentable, en l'état actuel des techniques.

D'autre part, le Conseil a limité sa décision aux techniques actuellement mises en œuvre en Nouvelle-Calédonie. En effet, l'instruction a permis d'établir que les travaux de recherches des substances se font par deux techniques de forage (l'une est la technique dite " air-core »; l'autre est le sondage carotté) dont il n'aurait pas été démontré qu'elles ont des incidences significatives sur l'environnement.

De telles limitations paraissent heureuses dans la mesure où la Nouvelle-Calédonie comme la Polynésie française possèdent, au niveau des sous-sols de leur domaine maritime respectif, un haut potentiel de présence d'hydrocarbures (pétrole, gaz) et de métaux (cobalt, cuivre, terres rares). Ces nouvelles ressources représentent des enjeux environnementaux et économiques importants et il apparaît évident que leur exploitation pourrait impacter la biodiversité des fonds marins. En effet, il existe plusieurs méthodes d'extractions pour exploiter ces ressources : le système de ramassage par bennes attachées à un câble et tractées par un navire, le système d'aspiration via un tube ou encore le système d'extraction par préleveurs libres et autonomes. Dans tous les cas, ces méthodes de ramassages impactent l'environnement marin. En effet, un rapport établi par le CNRS et I'IFREMER $\mathbf{4 8}$ indique qu'avec l'exploitation, l'habitat de certains animaux et micro-organismes pourrait être partiellement détruit. De même, le ramassage des terres rares par aspiration pourrait entraîner la disparition de plusieurs animaux et micro-organismes, en raison de l'action combinée de l'abrasion, de la décompression et en raison de l'augmentation de température dans le tube. Enfin, l'exploitation pourrait provoquer une augmentation de la concentration de composés toxiques dans le milieu marin.

Dans un tel contexte, il semble important que le Conseil constitutionnel ait laissé la porte ouverte à d'éventuelles questions prioritaires de constitutionnalité en matière minière.

En conclusion, il apparaît qu'une clarification du Conseil d'État sur les modalités de mise en œuvre de l'article 7 de la Charte de l'environnement dans les collectivités autonomes situées outre-mer s'impose. S'il semble que son arrêt du 13 février 2015 règle la situation s'agissant de la Polynésie française, la confusion introduite en Nouvelle-Calédonie par l'avis du Conseil d'État du 19 mai 2015 est fâcheuse. Un cadre juridique plus homogène en la matière serait donc salutaire.

48 Expertise scientifique collective, Impacts environnementaux de l'exploitation des ressources minérales marines profondes, Synthèse du rapport, CNRS-IFREMER, 2014. 